\title{
Extracting features for the linguistic variables of fuzzy rules using hidden Markov model
}

\begin{abstract}
In classifying handwritten characters, the stages prior to the classification phase play a role as major as the classification itself. This research work will be classifying the characters using a syntactical classification method namely fuzzy logic but will use the statistical method of Hidden Markov Model as an approach in extracting features for the linguistic variables of the fuzzy rule-based system. In this paper the feature extraction method will be highlighted and detailed. The HMM Model of a variable to be used in the classification system will be discussed. Experimental results from a few sample images show that the proposed technique is both effective and efficient to be used in extracting features for the linguistic variables of fuzzy rules.
\end{abstract}

Keyword: HMM model; Linguistic variable; Fuzzy logic; Handwritten character recognition 